BULL. AUSTRAL. MATH. SOC.

$08 A 40,06 \mathrm{Al} 2,08 \mathrm{~A} 60,20 \mathrm{MI} 4$

VOL. 27 (1983), 315-317.

\title{
POLYNOMIAL INTERPOLATION ON \\ UNIVERSAL ALGEBRAS
}

Peter Alexander Grossman

The thesis is concerned with the interpolation of functions on a universal algebra by means of polynomial functions. If $A$ is an algebra and $n$ is a positive integer, then the $n$-place constant functions and projections on $A$ generate the algebra $P_{n}(A)$ of $n$-place polynomial functions on $A$ (Lausch and Nöbauer [2]), while the projections alone generate the algebra $T_{n}(A)$ of $n$-place term functions on $A$ (Hule and Nöbauer [1]). The set of $n$-place functions on $A$ that coincide with some polynomial function on each subset of $A^{n}$ with at most $k$ elements is denoted by $L_{k} P_{n}(A)$. The set of $n$-place functions on $A$ that coincide with some polynomial function on each finite subset of $A^{n}$ is denoted by $L P_{n}(A) \cdot L_{k} T_{n}(A)$ and $L T_{n}(A)$ are defined analogously. It follows that

$$
L_{1} P_{n}(A) \supseteq L_{2} P_{n}(A) \supseteq \cdots \supseteq L P_{n}(A) \supseteq P_{n}(A)
$$

and

$$
L_{1} T_{n}(A) \supseteq L_{2} T_{n}(A) \supseteq \cdots \supseteq L T_{n}(A) \supseteq T_{n}(A)
$$

For each of these chains, the problem arises of finding which members of the chain coincide. A related question concerns the set $C_{n}(A)$ of $n$-place compatible functions (functions that preserve all congruences on $A):$ is it true that $C_{n}(A)=L_{2} P_{n}(A) ? \quad\left(C_{n}(A) \supseteq L_{2} P_{n}(A)\right.$ for any algebra $A$ and any $n$.) If $A$ is partially ordered, a similar question can be

Received 15 November 1982. Thesis submitted to Monash University, July 1982. Degree approved November 1982. Supervisor: Dr H. Lausch. 
asked about the set of $n$-place order-preserving functions on $A$. These problems form the subject matter of the thesis.

Chapter I contains a survey of results obtained by various authors. In the past, attention has been focused mainly on the problem of characterizing those algebras $A$ for which every $n$-place function on $A$ is in $L P_{n}(A)$.

Chapter II is concerned with a generalization of the chain problems stated above, where $P_{n}(A)$ or $T_{n}(A)$ is replaced by an arbitrary set $A$ of $n$-place functions on $A$. In parts of the chapter, $A$ is required to contain the $n$-place projections, and to be closed under an $n$-variable analogue of function composition. It is shown that, with these restrictions, the chain problem is equivalent to the $n$-place term function chain problem for some algebra.

In subsequent chapters, particular varieties of algebras are considered in detail with regard to the polynomial function and term function chain problems. Chapter III provides complete solutions to the chain problems for sets (algebras with no operations) and nullary algebras (algebras with only nullary operations). In Chapter IV, complete solutions to the chain problems are obtained for 1-unary algebras (algebras with one unary operation). In particular, for any 1-unary algebra $A$, $L_{3} P_{n}(A)=L P_{n}(A)$ for every $n, L_{2} T_{1}(A)=L T_{1}(A)$, and $L_{3} T_{n}(A)=L T_{n}(A)$ for every $n$. Some results are also obtained concerning 1-place compatible functions on 1-unary algebras.

Chapter $\mathrm{V}$ is concerned with the chain problems for semigroups. Particular attention is given to semilattices, for which a complete solution to the term function chain problem and a partial solution to the polynomial function chain problem are provided. There are also results on 1-place compatible functions and order-preserving functions on semilattices.

Semilattices of abelian groups are investigated in Chapter VI. The main result - that $L_{3} P_{1}(S)=L P_{1}(S)$ for any semilattice of abelian groups $S$ - is a generalization of a result of Hule and Nöbauer [1] for abelian groups. 


\section{References}

[1] Harald Hule and Wilfried Nöbauer, "Local polynomial functions on universal algebras", An. Acad. Brasiz. Ciênc. 49 (1977), 365-372.

[2] Hans Lausch and Wilfried Nöbauer, Algebra of polynomials (NorthHolland Mathematics Library, 5. North-Holland, Amsterdam, London; American Elsevier, New York, 1973).

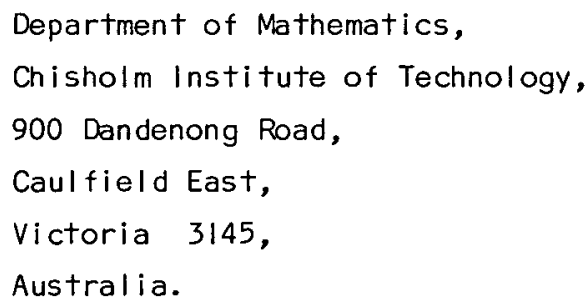

